\title{
LeGes
}

Ursula Münch

\section{COVID-19-Gesetzgebung in der Bundesrepublik Deutschland - zwischen Wahlkampf und Evidenzbasierheit}

\begin{abstract}
In akuten Krisen gerät das Spannungsverhältnis zwischen autonomieschonender und gemeinschaftsverträglicher Aufgabenbewältigung zusätzlich unter Druck: Die Nachfrage nach möglichst eindeutigen sowie transparenten Regelungen trifft auf die Notwendigkeit, so zügig wie möglich angemessen auf regionale und lokale Besonderheiten zu reagieren und dabei auch die wissenschaftlichen Erkenntnisse zu berücksichtigen. Diese Forderung nach Evidenzbasiertheit stellte im Zuge der Pandemie auch den deutschen Bundesstaat vor immense Herausforderungen, denen die Akteure häufig genug nicht gerecht werden konnten.
\end{abstract}

Beitragsart: Wissenschaftliche Beiträge der SGG-Tagung

Zitiervorschlag: Ursula Münch, COVID-19-Gesetzgebung in der Bundesrepublik Deutschland zwischen Wahlkampf und Evidenzbasierheit, in: LeGes 32 (2021) 3 


\section{Inhaltsübersicht}

1. Aufgabenverteilung im deutschen Bundesstaat

2. Gesetzgebung unter dem Druck der Inzidenzen

3. Politisches Handeln unter Unsicherheit

3.1. "Auf Sicht fahren»

3.2. Politische Entscheidungen unter den Bedingungen von Kontingenz

3.3. Wissenschaftliche Politikberatung

4. Interessens- und Zielkonflikte als Konstanten politischer Entscheidungsfindung

\section{Aufgabenverteilung im deutschen Bundesstaat}

[1] Alle föderalen Systeme kennen die «prekäre Balance» (von Beyme 2007, 209) zwischen «shared-rule» (Mitwirkungsrechte und Einflussmöglichkeiten subnationaler Akteure auf zentralstaatliche Entscheidungsprozesse) und «self-rule» (Ausmass je eigenständiger subnationaler oder zentralstaatlicher Entscheidungskompetenzen) (ElazAr 1994, XV). Grundsätzlich wird im «intrastaatlichen» Föderalismusmodell der Bundesrepublik die funktionale Aufgabenverteilung zwischen einer beim Zentralstaat angesiedelten Gesetzgebungskompetenz und einer bei den Ländern anzutreffenden Gesetzesausführung praktiziert. Die Aufgaben werden also überwiegend nach Funktionen - Gesetzgebung und Vollzug - und eher selten nach Staatsaufgaben organisiert. In Kombination mit dem deutschen Bundesratsmodell führt dies erstens zu einer ausgeprägten intrastaatlichen Beteiligung der 16 Landesregierungen an der Bundespolitik und zweitens zu einer interstaatlichen Kooperation sowohl auf der Ebene der Länder als auch zwischen Bund und Ländern. Bei der Unterscheidung zwischen «intrastaatlichem» und «interstaatlichem» Föderalismus, also zwischen föderalem Verbund- und Trennmodell, handelt es sich um eine Differenzierung von Idealtypen. Dass es in der Realität des Bundesstaates zu Überlappungen der Modelle kommt, lässt sich gerade auch für das föderale politische Handeln in Zeiten der Pandemie feststellen.

[2] Die Seuchenbekämpfung wird meist dem Katastrophenschutzrecht zugerechnet, das den deutschen Bundesstaat schon deshalb vor «besondere Herausforderungen» stellt, weil es sich durch ein «dichtes Normen- und Zuständigkeitsgeflecht auszeichnet», das «seine konkrete Gestalt oftmals keinem klaren Konzept als vielmehr politischen und geschichtlichen Zufälligkeiten verdankt» (Thiele 2012, 77-79). Gemäss Art. 74 Abs. 1 Nr. 19 Grundgesetz (GG) fällt die Seuchenbekämpfung in Deutschland unter die so genannte konkurrierende Gesetzgebungszuständigkeit des Bundes; sie umfasst unter anderem Massnahmen der Bekämpfung «gemeingefährlicher» oder übertragbarer Krankheiten. Die einzelnen Elemente der Seuchenbekämpfung beinhalten zweitens Massnahmen zum Erhalt bzw. zur Wiederherstellung der «öffentlichen Gesundheit» («Public Health»). In der COVID-Krise kommt dem «Gesetz zur Verhütung und Bekämpfung von Infektionskrankheiten beim Menschen» (Bundesinfektionsschutzgesetz, IfSG), mit dessen Erlass im Jahr 2000 das aus den 1950er und 1960er Jahren stammende und auf verschiedene Rechtsgrundlagen zerstreute Seuchenrecht umfassend novelliert worden war, vorrangige Bedeutung zu (Teichert 2015, 353). Das bundesdeutsche Infektionsschutzgesetz stellt den Teil des Gesundheitsrechts dar, bei dem nicht der einzelne Patient im Vordergrund steht, sondern die Prävention übertragbarer Krankheiten. Ziel ist es, die öffentliche Gesundheit auch durch unter Umständen massive Grundrechtseingriffe zu schützen (KIEssLING 2020). Durch das IfSG wurden nicht nur die Instrumentarien zur Erkennung und Überwachung übertragbarer Krankheiten neu strukturiert, sondern auch das Robert-Koch-Institut (RKI) als epidemiologisches Zentrum institutionalisiert. 
Vor der Pandemie war das bundesdeutsche Infektionsschutzgesetz durch eine Gestaltungsschwäche des Zentralstaats gekennzeichnet: Entsprechend der für den deutschen Verbundföderalismus typischen Aufgabenverteilung war (abgesehen von den Zuständigkeiten des Robert-KochInstituts) auch für den Krisenfall keine relevante Zuständigkeit des Bundes vorgesehen (MüNCH 2020). Der zuständige Bundesgesetzgeber war und ist entsprechend der bundesdeutschen föderalen Aufgabenverteilung auf die Ausführung des Gesetzes durch die Länder (und Gemeinden) in Form landeseigener Verwaltung angewiesen.

\section{Gesetzgebung unter dem Druck der Inzidenzen}

[3] Nachdem das Bundesgesetz in seiner ursprünglichen Fassung nicht auf Krankheitsausbrüche epidemischen Ausmasses zugeschnitten war, beschloss der Deutsche Bundestag im Frühjahr 2020, also zu Beginn der Pandemie, in grosser Eile weitreichende Gesetzesänderungen, u.a. neue Kompetenzzuweisungen an das Bundesgesundheitsministerium im Falle der in das Gesetz neu eingeführten «epidemischen Lage von nationaler Tragweite» in $\S 5$ Abs. 1 IfSG. Doch auch das im November 2020 in Kraft getretene «Dritte Gesetz zum Schutz der Bevölkerung bei einer epidemischen Lage von nationaler Tragweite», das zum einen die Voraussetzungen für eine solche Lage definierte und zum anderen im neu gefassten § 28a Abs.1 IfSG «besondere Schutzmassnahmen» aufzählt, schränkte die Möglichkeiten der deutschen Landesregierungen, landesspezifische Massnahmen zu erlassen, kaum ein. Nachdem die verschiedenen Gesetzesnovellen (HofmanN 2021) die grundsätzliche Aufgabenverteilung im Bundesstaat zunächst unberührt liessen, gewann etwas anderes an Bedeutung, nämlich die den bundesdeutschen Föderalismus ohnehin charakterisierende Kooperation der Exekutiven. Die im Grundgesetz nicht explizit vorgesehenen Besprechungen der Bundeskanzlerin mit den Ministerpräsidentinnen und -präsidenten der Länder wurden deutlich intensiviert. Doch auch im Rahmen dieser Beratungen der Regierungschefs der Länder und des Bundes (fälschlich als Ministerpräsidentenkonferenz (MPK) bezeichnet), gelang es selten, sich auf bundesweit verbindliche Kriterien für bestimmte Massnahmen zu einigen. Vielmehr spielte sich ein Ritual ein, das in Medien und Öffentlichkeit viel Kritik auslöste: Nach den Beratungen der Regierungschefinnen und -chefs, deren zu erwartende Ergebnisse meist schon im Vorfeld in den Medien diskutiert und kritisiert worden waren, dauerte es meist nur wenige Stunden, bis die erste Landesregierung bekannt gab, aus welchen Gründen sie von den getroffenen Vereinbarungen abweichen werde. Diese Tendenzen wurden dadurch begünstigt, dass die bundesdeutschen politischen Parteien sich bereits im Jahr 2020 auf die Bundestagswahl am 26. September 2021 einstellten. Vor allem der unionsinterne - bis April 2021 zunächst unerklärte - Wettstreit zwischen Armin Laschet (CDU), dem damaligen Ministerpräsidenten von Nordrhein-Westfalen, und dem Bayerischen Ministerpräsidenten Markus Söder (CSU) um die Position des Unionskanzler-Kandidaten weckte in der Öffentlichkeit den Eindruck, dass hier zwei Protagonisten den Verlauf der Pandemie und die Auseinandersetzung um die richtigen Massnahmen im Umgang mit COVID-19 zur persönlichen Profilierung nutzten. Während das Bund-Länder-Gremium MPK und vor allem die daran anschliessenden Pressekonferenzen mit Bundeskanzlerin Merkel also zur parteipolitischen Profilierung genutzt wurden, blieb der genuin föderale Aspekt dieses Wettbewerbs ungenutzt: Man vergab die Möglichkeit, den Erfolg der verschiedenen Massnahmen zur Eindämmung der Pandemie durch einen Vergleich von Landesmassnahmen und Landesinzidenzen zu testen und einen empirisch begründbare Erprobung unterschiedlicher Eingriffsformen anzustellen. 
[4] Aufgrund massiver öffentlicher Kritik an Form und Inhalt der Beschlussfassungen durch die MPK wurde schliesslich durch eine - ebenfalls sehr umstrittene - bundesgesetzliche Regelung des 4. Bevölkerungsschutzgesetzes vom 22. April 2021 die «Bundesnotbremse» erlassen: Ab einer stabilen Inzidenz von 100 Neuinfektionen pro 100'000 Einwohner in den letzten sieben Tagen in einem Landkreis (entscheidend waren die dem RKI gemeldeten Zahlen) griff bundesweit eine einheitliche «Notbremse» im Sinne bundesweit einheitlicher Schutzmassnahmen. Diese Regelung war im gesamten Bundesgebiet vom 23. April bis Ende Juni 2021 in Kraft.

[5] In einer späteren Phase der Pandemie, nach Entwicklung und Verbreitung der COVID-Impfstoffe, bestand in Deutschland grosse Unsicherheit und sichtbarer Dissens über die künftigen Massstäbe der bundesdeutschen Pandemieeindämmungspolitik: Der damalige Bundesminister für Gesundheit, Jens Spahn (CDU) stellte im August 2021 fest, die «50er Inzidenz im Gesetz» habe angesichts der Impfquote in der Bevölkerung «ausgedient», der «neue Parameter» sei die «Hospitalisierung». Dieses Umsteuern des Bundesgesundheitsministers war ein Zugeständnis vor allem an die Skeptiker in der CDU/CSU-Bundestagsfraktion gegenüber der von der damaligen Bundesregierung aus CDU/CSU und SPD gewünschten Verlängerung der «epidemischen Lage» gemäss $\S 5$ Abs. 1 S. 1 IfSG. Der Präsident des dem Bundesgesundheitsministerium unterstellten RKI vertrat hingegen eine andere Auffassung: Er plädierte dafür, die Inzidenz weiterhin als zentrale Grösse heranzuziehen; sie sei für die Politik erforderlich, um rechtzeitig Gegenmassnahmen initiieren zu können. Laut Bundesgesundheitsministerium gilt die Hospitalisierungsinzidenz als ein «wichtiger Parameter, um das Infektionsgeschehen zu beurteilen - aber nicht der Einzige».

\section{Politisches Handeln unter Unsicherheit}

[6] Im Zuge der Pandemiebekämpfung wurde es immer wieder erforderlich, ohne ausreichende Informationen, zum Beispiel über die Ansteckungswege von COVID-19, kurzfristig politische Entscheidungen zu treffen. In allen Phasen der bundesdeutschen Pandemieeindämmungspolitik lautete das Schlagwort von Bundes- und Landespolitikerinnen und -politikern, angesichts der vielen unbekannten Grössen von COVID-19, es gelte «auf Sicht» zu fahren. Mittel- oder gar langfristig angelegte Szenarien und Leitlinien für politisches Handeln wurden unter anderem von den Medien zwar angemahnt, eine entsprechende politische Festlegung galt angesichts der verschiedenen Unwägbarkeiten den meisten Verantwortlichen aber als viel zu gewagt (Lово 2021).

\section{1. «Auf Sicht fahren»}

[7] So wurde zum Beispiel anfänglich von bundesdeutschen politischen Akteuren Zweifel am Sinn des Tragens medizinischer bzw. sog. Alltagsmasken geäussert. Die entsprechenden eher skeptischen Stellungnahmen u.a. von Bundeskanzlerin Angela Merkel dürften aber vor allem auf den eklatanten Mangel an medizinischen Schutzmasken zurückzuführen gewesen sein. Während einige deutsche Länder zunächst noch eine Maskenpflicht ablehnten, wurde Ende April 2020 schliesslich das Tragen eines Mund- und Nasenschutzes (sog. Alltagsmasken) empfohlen. Ebenfalls auf der Grundlage von anfänglich grosser Unsicherheit entschieden die Landesregierungen über die Reichweite der sog. «Lockdown»-Regelungen. Als erstes deutsches Land erliess die Bayerische Staatsregierung am 24. März 2020 (gültig bis 19. April 2020) per Verordnung strenge Ausgangsbeschränkungen. Die eigene Wohnung durfte nur verlassen, wer einen trifti- 
gen Grund hatte. Der Freistaat ging mit seiner Regelung über den vereinbarten Bund-LänderBeschluss hinaus, der empfohlen hatte, im Freien nur noch ein Treffen von maximal zwei Personen (davon eine haushaltsfremde) zu erlauben. Die spezifische bayerische Regelung hatte in der deutlich verspäteten Hauptsacheprüfung des Bayerischen Verwaltungsgerichtshofs keinen Bestand (VGH MüNCHEN 2021) und wurde wegen eines Verstosses gegen das Übermassverbot nachträglich für unwirksam erklärt. Der Staatsregierung habe aufgrund der unsicheren Lage zwar ein Einschätzungsspielraum zugestanden, es wären aber - so der VGH - weniger belastende Eingriffsmöglichkeiten wie etwa Kontaktbeschränkungen im öffentlichen Raum zur Verfügung gestanden.

\subsection{Politische Entscheidungen unter den Bedingungen von Kontingenz}

[8] Als ein zentrales Problem moderner Politik gilt der Umstand, dass die Moderne einen materiell, kulturell, kognitiv und historisch «neuartigen Möglichkeitshorizont des Entscheidens und Handelns» konstituiert; dieser rechtfertige es, «gerade die politische Entscheidbarkeit als zentrales Charakteristikum von Gegenwartsgesellschaften zu verstehen» (GReven 2000, 24). Politische Entscheidungen unterscheiden sich von Willensbekundungen im Alltag nicht nur durch ihren Grad an Allgemeinverbindlichkeit, sondern auch dadurch, dass sie zum einen den Spielraum weiterer Entscheidungen einengen und zum anderen auch über den Handlungsraum anderer bestimmen (Rüв 2012,117). Nicht nur, aber vor allem im Zuge einer Pandemie, müssen diese politischen Festlegungen zwangsläufig unter den Bedingungen von Kontingenz getroffen werden; Kontingenz verstanden als der ambivalente Bereich des Möglichseins und des «auch-anders-möglichSeins». Entscheidungen sind möglich, aber keine einzelne von ihnen ist zwingend so notwendig. Politik beruht nicht auf ausserpolitischen Fundamenten und nicht auf letzten Gründen und notwendigen Wahrheiten. Vielmehr muss Politik als ein offener Möglichkeitsraum gefasst werden, bei dem es immer um Abwägungen und um Entscheidungen zwischen mehreren Möglichkeiten geht. Die damit einhergehende grundsätzliche Offenheit konfrontiert die politisch Verantwortlichen gerade in einer digitalen Mediengesellschaft mit dem durchaus gegenläufigen Anspruch der Öffentlichkeit, die jeweiligen Festlegungen sowie die Entscheidungen und Nicht-Entscheidungen darzulegen und vor dem Hintergrund der faktischen Lage zu begründen.

[9] Auch wenn Einigkeit darüber besteht, dass eine umfassende Darlegung von Entscheidungskriterien für einen rationalen Diskurs wünschenswert wäre, steht gleichzeitig ausser Frage, dass sowohl die Notwendigkeit der Gefahrenabwehr als auch das zumindest anfängliche Nichtwissen über die Gesetzmässigkeiten des Infektionsverlaufs im Verlauf der COVID-Krise dazu führten, dass politische Entscheidungen auf der Grundlage unzureichender empirischer Daten getroffen werden mussten. Obwohl das Politikfeld öffentliche Gesundheit massgeblich von den Erkenntnissen und Fortschritten der Wissenschaft abhängig ist, ändert dies nichts an der eigenen spezifischen Logik von Politik: Nach geeigneter Abwägung sind allgemeinverbindliche Entscheidungen über die öffentlichen Angelegenheiten zu fällen. Während der erste umfassende «Lockdown» zu Beginn der Pandemie im Frühjahr 2020 bei ca. 75 \% der bundesdeutschen Bevölkerung auf breite Zustimmung stiess (ARD-Deutschland-Trend 2020a), provozierte die spätere allmähliche Lockerung von Massnahmen angesichts der als «ungerecht» empfundenen Widersprüchlichkeit von Detailregelungen, z.B. zur Öffnung bzw. Schliessung im Einzelhandel, viel Kritik. 


\subsection{Wissenschaftliche Politikberatung}

[10] Zu Beginn der Pandemie war die Nachfrage in der bundesdeutschen Öffentlichkeit nach politischer Handlungsstärke gross. Eine gewisse Sehnsucht in der Bevölkerung nach exekutiver Autorität jenseits des Pluralismus der Parteien und der unterschiedlichen Interessenlagen war unverkennbar: Acht von zehn Befragten vertraten im November 2020 die Einschätzung, strenge Corona-Regeln seien Voraussetzung, um die Corona-Krise in den Griff zu bekommen; nur jeder oder jede Fünfte war gegenteiliger Auffassung (ARD-Deutschland-Trend 2020b). Die Haltung gegenüber wissenschaftlicher Politikberatung war demgegenüber ambivalent: Während einerseits ein sehr grosses öffentliches Interesse an den Erkenntnissen der einschlägigen Fachwissenschaften bestand, wurde in der Öffentlichkeit auch der Vorwurf erhoben, sowohl Regierungen als auch die Leitmedien hätten im Zuge der Pandemieeindämmung vor allem die wissenschaftliche Expertise angefragt, deren Positionen und Lösungsvorschläge am besten zum ohnehin eingeschlagenen politischen Kurs passten (Weingart 2021, 29). Die damalige deutsche Bundeskanzlerin Angela Merkel (CDU) hatte diesem Vorwurf widersprochen: «Nicht nur die, die jetzt gerade eingeladen sind, sind diejenigen, mit denen ich spreche, mit deren Ergebnissen ich mich befasse». Sie selbst, so die Bundeskanzlerin weiter, verfolge alle Meinungsbildungen und sei «nicht ignorant». Die Wissenschaftler, ihre Beraterrunde, seien sich auch gar nicht alle einig. Allerdings habe sie selbst vor dem Hintergrund des Risikos einer schweren Erkrankung auch junger Menschen eine politische Entscheidung getroffen. Und zwar die Entscheidung gegen den Versuch, eine Herdenimmunität zu erreichen. Deshalb lade sie Wissenschaftler, die dazu raten würden, auch nicht zu Gesprächen ein (BAYerischer Rundfunk 2021).

[11] Damit sprach Angela Merkel zutreffend das sog. "false balance»-Problem an, also das Problem falscher Ausgewogenheit: Eine wissenschaftliche Minderheitsmeinung oder sonstige abwegige Auffassungen sollten nicht durch vermeintlich ausgewogene pro-/contra-Einladungen "geadelt» werden (GrüNINGER 2021). Sowohl COVID-19 als auch die Klimakrise führen vor Augen, dass eine «Epistemisierung des Politischen unter demokratietheoretischen Gesichtspunkten» (BoGNer 2021) nicht wünschenswert ist. "Gute Politik» lasse sich nicht (oder nur selten) auf die richtige Lösung von Wissensfragen reduzieren, und politische Konflikte liessen sich auch nicht mit dem Verweis auf wissenschaftliche Wahrheiten befrieden.

[12] Insgesamt erscheint das Verhältnis von Politik und Wissenschaft in der digitalen Mediengesellschaft sehr spannungsgeladen: Beobachten wir zum einen eine steigende Abhängigkeit der Politik von wissenschaftlichem Sachverstand, sind gleichzeitig wachsende Vorbehalte in Teilen der Bevölkerung gegenüber einer Beeinflussung der Politik durch Wissenschaftler wahrzunehmen, die sich zum Teil auch in wissenschaftsskeptischer oder sogar wissenschaftsfeindlicher Haltung äussern. Die entsprechenden Vorbehalte werden die digitale Zugänglichkeit zu kollaborativen Wissensspeichern (etwa Wikipedia) sowie die Erleichterung der Vernetzung und damit der Verbreitung von Falschinformationen geschürt (Pantenburg et al. 2021). In dem Masse, in dem wissenschaftliche Expertise zwischen die (politischen) Fronten gerät und eine entsprechende Polarisierung von der Öffentlichkeit wahrgenommen wird, wird die Unabhängigkeit von Wissenschaftlern angezweifelt: Zu jeder Expertise lässt sich (vermeintlich) Gegenexpertise mobilisieren. In der Folge geraten die staatlichen Bemühungen, das politische Handeln evidenzbasiert auszurichten, zusätzlich unter Druck: Zum Problem des Handelns unter den Bedingungen von Unsicherheit und Kontingenz tritt die Herausforderung, die Überlegenheit der vorhandenen wissenschaftlichen Erkenntnisse gegenüber «emotionalen Beweisführungen» einer «kritischen» 
Bürgerschaft rechtfertigen und deren selbstermächtigender Freude am Gegenwissen an der Rolle des eigentlichen Experten begegnen zu müssen (Pantenburg et al. 2021, 24).

[13] Angesichts dieses Wettstreits von politisch Verantwortlichen sowie den Kritikern von Coronamassnahmen, sich in Sachen wissenschaftlicher (oder eben auch halbwissenschaftlicher) Erkenntnis zu übertrumpfen, gerät in Vergessenheit, dass es letztlich darum geht, politische Entscheidungen treffen und begründen zu müssen. Auch in der Krise - egal ob der Klimakrise oder der COVID-19-Krise - bleibt der Primat der politischen Willensbildung und Entscheidungsfindung also bestehen. Damit ist nicht gemeint, dass die politisch Verantwortlichen nicht die wissenschaftlichen Erkenntnisse zur Kenntnis zu nehmen und in ihre Entscheidungen einzubeziehen haben. Diese Feststellung zielt vielmehr darauf ab, dass Wissenschaft einer anderen Logik folgt als Politik. Wissenschaft kann daher der Politik nicht die Aufgabe abnehmen, allgemeinverbindliche politische Entscheidungen zu treffen und dafür Mehrheiten herzustellen.

[14] Dass die Evidenzbasiertheit politischer Entscheidungen auch durch Defizite in der Datenerhebung leidet, wurde in der Bundesrepublik Deutschland zuletzt beim Stand der Durchimpfung der Bevölkerung sichtbar. Obwohl die Impfquote ein zentrales Kriterium für die Lockerung von grundrechtsrelevanten Massnahmen der Pandemieeindämmung darstellt, wurde im Sommer 2021 bekannt, dass die Datenlage bezüglich des Impfstatus der Wohnbevölkerung in Deutschland ausgesprochen lückenhaft ist: Ein Vergleich der im Digitalen Impfquotenmonitoring (DIM) zusammengefassten Angaben und einer weiteren Erhebung des RKI, dem auf Befragungen basierenden Impfmonitoring «Covimo» brachte eine nennenswerte Diskrepanz zum Vorschein (Robert-Косн-Institut 2021). Diese beruht offenbar darauf, dass die entsprechenden Meldungen der Impfzentren, Krankenhäuser sowie der mobilen Impfteams relativ zügig und vollständig erfasst werden, während die von Betriebsmedizinern sowie niedergelassenen Ärztinnen bzw. Privatärzten vorgenommenen Impfungen offenbar verzögert und wohl auch nicht vollständig erfasst wurden. Wie bereits zu Beginn der Pandemie, wird in diesem Zusammenhang erneut die Leistungsfähigkeit des deutschen kommunalen Öffentlichen Gesundheitsdienstes mit seinen ca. 375 kommunalen Gesundheitsämtern kritisiert: Wurden anfänglich die Infektionszahlen mit Verzögerung und Lücken an das RKI gemeldet, wird mit Blick auf die Dokumentation der Impfungen bemängelt, dass das Ziel, den Bearbeitungsaufwand niedergelassener Ärzte zu reduzieren, offenbar zum verwirrenden Nebeneinander unterschiedlicher Übermittlungsformate führte.

\section{Interessens- und Zielkonflikte als Konstanten politischer Entscheidungsfindung}

[15] $\mathrm{Zu}$ den Besonderheiten der bundesdeutschen Pandemiebekämpfung gehört ihre Überlagerung durch die parteipolitische Personalisierung und den Bundestagswahlkampf. So wurden die Entscheidungen der Ministerpräsidentenkonferenz von Medien und Öffentlichkeit immer auch unter dem Vorzeichen des Wettbewerbs innerhalb von CDU und CSU um die UnionsKanzlerkandidatur beurteilt.

[16] Darüber hinaus wird die stringente Ausrichtung der COVID-19-Gesetzgebung bzw. entsprechender Massnahmen an Evidenzen durch zusätzliche Faktoren beeinträchtigt: Nicht nur in Deutschland ist eine wachsende Skepsis gegenüber wissenschaftlicher Politikberatung zu beobachten, die durch populistische Anti-Establishment-Argumentation zusätzlich geschürt wird. 
Zudem ist bei der Forderung nach einer stärker evidenzorientierten Politik zu berücksichtigen, dass es auch bei der Pandemiebekämpfung divergierende Interessen sowie Zielkonflikte gibt. So sollte die Schwere der Pandemie nicht darüber hinwegtäuschen, dass es auch in einer grossen Gesundheitskrise politische Zielkonflikte geben kann, die sich zwangsläufig auf die Prioritätensetzung in der Pandemieeindämmungspolitik auswirken.

[17] Eine andere Frage ist, ob und wie über die Existenz dieser Zielkonflikte öffentlich debattiert wird (DАвRоск 2021). Auch in der bundesdeutschen Politik wurde kontrovers darüber diskutiert, ob und wie Lebensschutz einerseits und Freiheitsrechte bzw. wirtschaftliche Perspektiven und Bildungschancen andererseits gegeneinander abzuwägen seien. Der Bayerische Ministerpräsident Markus Söder (CSU), aber auch Bundeskanzlerin Angela Merkel vertraten insgesamt die Position, Politik habe die Aufgabe, jedes Leben zu schützen und wenn möglich auch zu retten. Dieser Haltung widersprach nicht zuletzt der damalige Präsident des Deutschen Bundestages, Wolfgang Schäuble (CDU): «Dem Schutz von Leben in der Coronakrise kann nicht alles untergeordnet werden». Unklar blieb bei dieser Auseinandersetzung die Bestimmung des Massstabes: Ging es dem Bayerischen Ministerpräsidenten und der Bundeskanzlerin vor allem um die Verhinderung einer Überlastung des medizinischen Systems? Oder plädierten sie für eine in ihrer Reichweite deutlich weitergehende Verpflichtung des Staates, Individuen auch vor solchen Gefahren zu schützen, die von Seite Dritter drohen? Der letztgenannte Fall ginge mit einem problematischen Übergang von der Gesamtbetrachtung zur Individualperspektive einher: Dann könnte die Pandemiebekämpfung bis zum völligen Verschwinden des Virus per definitionem nie abgeschlossen sein; Massnahmen zur Pandemiebekämpfung wären in diesem Fall und bei dieser Interpretation «zeitlich wie sachlich unbeschränkt rechtlich zulässig, wenn nicht sogar geboten» (HeINIG et al. 2020, 863). Beide Positionen lassen sich begründen und mögen legitim erscheinen. Welcher der beiden man den Vorrang gibt, lässt sich jedoch nicht wissenschaftlich begründen. Hierbei handelt es sich vielmehr um eine (zu begründende) politische Entscheidung.

[18] Im Zuge der Pandemieeindämmungspolitik musste nicht nur die Politik «auf Sicht fahren», sondern auch der so dringend benötigte wissenschaftliche Rat wurde vor allem anfänglich «häufig auf wackeligen Grundlagen gegeben». Man habe es nicht geschafft, «überregionale, differenzierte Informationen zu bekommen. Zumindest nicht in dem Ausmass, das wir bräuchten» (Satyajit Rath nach Pfeifer 2021). Um diesen strukturellen Mängeln zu begegnen, ist eine interdisziplinäre und beständige Struktur der wissenschaftlichen Politikberatung unerlässlich. Diese kann in Zeiten der «Normalität» Vertrauen aufbauen, das dann in Zeiten der Krise eine stärkere Legitimation von Politikberatung bewirken kann. Ein Beispiel für entsprechende Strukturen bietet die «Scientific Advisory Group for Emergencies» (SAGE) im Vereinigten Königreich. Ein anderer Ansatz stellt eine Stärkung der Weltgesundheitsorganisation (WHO) dar.

[19] Einigkeit besteht, dass nach weltweiter Eindämmung der Pandemie eine komparative Evaluation der verschiedenen Erscheinungsformen einer Pandemie und der unterschiedlichen Bewältigungsstrategien unerlässlich sein wird.

[20] Sowohl bei der Pandemie als auch bei der Erderwärmung handelt es sich um sog. «Krisen eines neuen Typus» (Wolfgang Merkel), die uns Verschiedenes vermitteln: Auch die wissenschaftliche Fundierung von Politik, also das Streben nach evidenzbasierter Gesetzgebungstätigkeit, ändert nichts an der Existenz von Interessenkonflikten. Politische Akteure haben auf der Grundlage ihrer parlamentarischen Mehrheit im repräsentativen System der Bundesrepublik Deutschland allgemeinverbindliche Entscheidungen zu treffen, für die sie die politische Verantwortung tragen. Die Wissenschaften können beraten und helfen, das «Fahren auf Sicht» möglichst unfallfrei 
zu gestalten. Das entbindet aber nicht davon, die immer möglichen politischen Alternativen abzuwägen.

[21] Die Nähe von Wissenschaft und Politik in der Pandemie und die Fähigkeit der Wissenschaft, akute Krisen lösen zu helfen, nährte die Vorstellung, das sei die wesentliche oder sogar alleinige Aufgabe von Wissenschaftlerinnen und Wissenschaft. Von dieser Fehleinschätzung aus wäre es nur ein «intellektueller Katzensprung» (SPEISER 2021, 375) zur Idee, vor allem solche Wissenschaften zu fördern, von denen man sich die Eignung zur Krisenlösung verspricht. Das würde nicht nur den gesellschaftlichen Blick einschränken, sondern auch den Fluss und die Vielfalt der Finanzströme. Eine derartige Einschränkung führte «paradoxerweise dazu, dass man sich mittelfristig der Krisenlösungsfähigkeit beraubt, die man beleben wollte» (SPEISER 2021, 375).

Prof. Dr. URsula MüNCh, Akademie für Politische Bildung / Universität der Bundeswehr München.

\section{Literaturangaben}

ARD-Deutschland-Trend März Extra (2020a) https://www.infratest-dimap.de/umfragen-analysen/bundesweit/ ard-deutschlandtrend/2020/maerz-extra/ [14.10.2021].

ARD-Deutschland-TRend extra "Coronamassnahmen» (2020b) https://www.infratest-dimap.de/umfragen-analysen/ bundesweit/ard-deutschlandtrend/2020/november-extra-coronamassnahmen/ [14.10.2021].

BAyerischer Rundfunk: Corona-Experten: Lässt sich die Kanzlerin einseitig beraten? https://www.br.de/nachrichten/ deutschland-welt/corona-experten-laesst-sich-die-kanzlerin-einseitig-beraten,SN5NgG3 [14.10.2021].

Beyme von, Klaus (2007): Föderalismus und regionales Bewusststein. Ein internationaler Vergleich, München.

Bogner, Alexander (2021): Die Epistemisierung des Politischen. Wie die Macht des Wissens die Demokratie gefährdet, Stuttgart.

Dabrock, Peter (2021): «Not kennt kein Gebot»? Ethische Perspektiven der Pandemie-Bekämpfung, in: Aus Politik und Zeitgeschichte Heft 24-25, S. 4-10 https://www.bpb.de/shop/zeitschriften/apuz/334630/medizin-und-ethik-in-derpandemie [14.10.2021].

Elazar, Daniel J. (Hrsg.) (1994): Federal systems of the world: a handbook of federal, confederal and autonomy arrangements, London (2. Aufl.).

Greven, Michael Th. (2000): Kontingenz und Dezision. Beiträge zur Analyse der politischen Gesellschaft, Wiesbaden.

Grüninger, Servan Luciano (2021): «False Balance» in den Medien: Was wissenschaftlich stimmt, ist keine Frage der Mehrheitsmeinung, in: Reatch (Research. Think. Change) vom 7.9.2021 https://reatch.ch/publikationen/false-balancein-den-medien-was-wissenschaftlich-stimmt-ist-keine-frage-der-mehrheitsmeinung [14.10.2021].

Heinig, Hans Michael / Kingreen, Thorsten / Lepsius, Oliver / Möllers, Christoph / Volkmann, Uwe / Wissmann, Hinnerk (2020): Why Constitution Matters - Verfassungsrechtswissenschaft in Zeiten der Corona-Krise, in: JuristenZeitung 75, Heft 18, S. 861-872.

Hofmann, Hans (2021): Das «Corona-Recht» - Zwischen verfassungsgemäßer Rechtsetzung und operativ-notwendiger Krisenreaktion, in: Zeitschrift für Gesetzgebung 2/2021, S. 109-129.

Kiessling, Andrea (2020): Coronavirus, Masern und die Grundrechte, in: Verfassungsblog vom 2.3.2020 https://verfassungsblog.de/coronavirus-masern-und-die-grundrechte/ [14.10.2021].

Lово, Sascha (2021): Verfahren auf Sicht, in: Spiegel online vom 24.2.2021 https://www.spiegel.de/netzwelt/ netzpolitik/angela-merkel-in-der-corona-pandemie-verfahren-auf-sicht-kolumne-a-4587b945-d861-4325-86d5ca19f64440d2 [14.10.2021]. 
MüNCH, URsula (2020): Wenn dem Bundesstaat die Stunde der Exekutive schlägt: der deutsche (Exekutiv-) Föderalismus in Zeiten der Coronakrise, in: Jahrbuch des Föderalismus 2020. Föderalismus, Subsidiarität und Regionen in Europa. Hrsg. vom Europäischen Zentrum für Föderalismus-Forschung Tübingen, Baden-Baden, S. 209-225.

Pantenburg, Johannes / Reichardt, Sven / Sepp, Benedikt (2021): Corona-Proteste und das (Gegen-)Wissen sozialer Bewegungen, in: Aus Politik und Zeitgeschichte (APuZ Heft 3-4, S. $22-27$ https://www.bpb.de/shop/zeitschriften/apuz/ 325615/wissen [14.10.2021].

Pfeifer, David (2021): «Sprechen Sie bitte nicht immer von Wellen». Immunologe Satyajit Rath im Interview, in: Süddeutsche Zeitung vom 28./29.8.2021 https://www.sueddeutsche.de/politik/coronavirus-pandemie-indienimmunologie-1.5392554 [14.10.2021].

Robert-Косн-Institut (2021): COVID-19 Impfquoten-Monitoring in Deutschland (COVIMO). Report 6 vom 10.8.2021 https://www.rki.de/DE/Content/InfAZ/N/Neuartiges_Coronavirus/Projekte_RKI/COVIMO_Reports/covimo_studie_ bericht_6.pdf?__blob=publicationFile [14.10.2021].

RüB, Friedbert W. (2012): Politische Entscheidungsprozesse, Kontingenz und demokratischer Dezisionismus. Eine policyanalytische Perspektive, in: Toens, Katrin / Willems, Ulrich (Hrsg.): Politik und Kontingenz, Wiesbaden, S. 117-142.

SPeiser, Guido (2021): Die Krise verwischt den Grenzbereich, in: Forschung und Lehre 5/21, S. 374-375.

Teichert, Ute (2015): Der öffentliche Gesundheitsdienst, in: Thielscher, Christian (Hrsg.): Medizinökonomie 1., Das System der medizinischen Versorgung, 2. Auflage, Wiesbaden, S. 351-372.

Thiele, Alexander (2012): Katastrophenschutzrecht im deutschen Bundesstaat, in: Härtel, Ines (Hrsg.), Handbuch Föderalismus - Föderalismus als demokratische Rechtsordnung und Rechtskultur in Deutschland, Europa und der Welt, Band 3: Entfaltungsbereiche des Föderalismus, Heidelberg, S. 69-108.

Verwaltungsgerichtshof München (2021): Beschluss vom 4.10.2021 https://www.gesetze-bayern.de/Content/ Document/Y-300-Z-BECKRS-B-2021-N-29086 [14.10.2021].

Weingart, Peter (2021): Wissenschaftliche Politikberatung in Krisenzeiten, in: Aus Politik und Zeitgeschichte (APuZ) Heft 3-4, S. 28-32 https://www.bpb.de/shop/zeitschriften/apuz/325615/wissen [14.10.2021]. 